\title{
Truth-telling and Respect for Autonomy
}

\author{
Maximilian Kiener \\ This is the accepted but pre-proofs version. The final version is published in (2018) The American
} Journal of Bioethics (Neuroscience) 9 (3), 193-194.

Gligorov (2018) claims that placebo treatment for pain is consistent with respect for patient autonomy. She supports this view by means of empirical evidence on how placebos work as well as an ethical argument about the relation between truth-telling and respect for autonomy. When focusing on empirical evidence, Gligorov surveys studies which purport to show that the administration of placebos and known analgesics often "recruit the same biological mechanisms" (9): when physicians administer placebos and thereby create positive expectations in the patient, this can affect brain regions in the same way as known analgesics do. Therefore, Gligorov concludes, the physician who administers a placebo by saying 'I am giving you a pill that will relieve your pain' makes a true statement. In her ethical argument, Gligorov claims that since the aforementioned physician's statement is true, "informing the patient that the clinician expects that taking this pill will treat the pain would not be misleading" (10), and it would also not be deceiving as "[d]eception requires departure from the truth in a way that does not exist in the context of placebo analgesia" (10). On these grounds, Gligorov argues that placebo treatment for pain, even if not explicitly labeled as such, is consistent with respect for patient autonomy.

Gligorov's paper is to be applauded for its contribution to understanding the nature of pain and challenging an oversimplified dichotomy between placebos and known analgesics. However, as I shall argue, Gligorov is mistaken in assuming that truth-telling guarantees respect for autonomy. I will claim that the physician's adherence to norms of trust provides a test for his respect of patient autonomy, and as Gligorov's proposal for the use of placebos fails this test, it fails to respect patient autonomy. I conclude by outlining the conditions under which administering placebos according to Gligorov's proposal would also invalidate the patient's informed consent. Throughout my commentary, I will focus on Gligorov's ethical arguments only. I will not challenge her empirical claims or the conclusion she draws from them, namely, that the biological mechanisms in the brain triggered by known analgesics and by placebos may be the same, and hence a physician administering a placebo makes a true statement in saying 'I am giving you a pill that will relieve your pain.' 


\section{Truth, Trust, and Autonomy}

Trust is the disposition to rely on another person's behavior beyond one's ability of surveillance. So understood, trust is integral to a functioning patient-physician relationship. It facilitates co-operation between patient and physician, without which promoting the patient's health would be impossible. However, over and above its general significance, trust is specifically important for patients. The complexity of medicine makes it impossible for patients to verify everything that is important to them. Patients have to trust their physician and the information provided by him if they are to exercise their autonomy, i.e. make reasoned and informed decisions about which medical procedures to undergo or which drugs to take. Hence, for patients, trust is of great instrumental value for the exercise of their autonomy. By the same token, however, a focal element in patients' autonomous decisionmaking lies with the physician: whether patients actually make the reasoned and informed decision they intend to make depends on the provision of appropriate information by the physician, i.e. it depends on whether the physician responds appropriately to patients' trust. Due to these links between trust and autonomy, a physician's violations of norms of trust, e.g. by misleading or deceiving a patient in the provision of information, adversely affect a patient's decision-making process and are therefore also violations of respect for patient autonomy. Hence, in the context of the patient-physician relationship, the physician's adherence to norms of trust provides a test for his respect of patient autonomy.

One central norm of trust is what I shall call the proviso of hypothetical transparency. Even though a trusting person refrains from "the precise spelling out of terms" (Greenspan 2003 , 161) which the trusted person may operate on, trust always comes with the proviso that the trusted party's relevant actions as well as "the motives of the trusted party have to be able to be made transparent to the one who extends trust" (Ibid.). This proviso is respected only if full transparency did not lead to the trusting person being justified in feeling resentment or insulted. As Greenspan claimed, "appropriate trust is trust that would survive a certain kind of knowledge on the part of the person who extends trust" (Ibid.).

Gligorov's account on the use of placebo violates the proviso of hypothetical transparency and therefore also fails to respect patient autonomy. A patient would indeed be justified in feeling resentment or insulted if the administration of a placebo were made fully transparent to him after he had received it. This is because, contrary to what Gligorov claims, Gligorov's proposed use of placebos misleads and deceives patients. Gligorov's two claims, namely (i) "informing the patient that the clinician expects that taking this pill will treat the pain would not be misleading" (10) because the statement is true, as well as (ii) that 
"[d]eception requires departure from the truth" (10), are simply not correct. There are several ways of misleading and deceiving someone by means of true statements. For instance, suppose you want to buy a car from me and you ask me whether there are problems with the air conditioning. I answer that I drove the car on a hot day and did not have any problems. Let us assume this is a true statement. However, it can still be misleading and deceptive because I may be talking about a day ten years ago, while you reasonably assume that it must have been a recent event. I misled and deceived you without saying anything that was literally false (similar examples are prevalent in the literature on deception. See for instance Carson 2010, 55). The same holds in cases of placebo treatment. The statement 'I am giving you a pill that will relieve your pain' is true but ambiguous. As Gligorov herself admits, it can mean either pain relief due to the pharmaceutical effects as observed in known analgesics, or pain relief due to the way in which the pill is administered and positive expectations are created. As receiving known analgesics is the expected treatment, the patient will likely acquire the false belief that that is what he has been given, and that its effect will not depend primarily on the patient-physician interaction and the creation of positive expectations. The combination of ambiguity and the reasonableness of falsely believing that one has been given a known analgesic makes the statement 'I am giving you a pill that will relieve your pain' misleading. And the fact that the physician either intends or at least foresees that the patient will acquire a false belief about his treatment makes the statement deceptive. By misleading and deceiving the patient in this way, the physician fails to treat the patient as someone who is entitled to know how his pain relief is supposed to be achieved. The physician ceases to address the patient as a rational individual but rather uses conversation as a causal lever, i.e. the physician uses words to cause expectations that will then relieve pain. On these grounds, the patient would indeed be justified in feeling resentment and insulted if the behavior of the physician were made fully transparent to him after the administration of placebo treatment. Therefore, the physician's behavior violates the proviso of hypothetical transparency and thereby also disrespects the patient's autonomy. Hence, pace Gligorov, truth-telling does not guarantee respect for autonomy.

\section{Ambiguity, Ignorance, and Consent}

The result that Gligorov's proposal fails to respect patient autonomy not only casts doubt on the ethical permissibility of placebos in general, but also, more specifically, substantiates concerns about the validity of the patient's consent to placebo treatment. As O'Neill correctly pointed out, "consent to a procedure under some description does not entail consent even to 
equivalent or entailed descriptions of the same act" (O'Neill 2002, 155). So, even if the physician's statement about 'a pill that will relieve pain' is true, this is not sufficient to ensure the validity of the patient's consent to placebo treatment. If it is not evident to the patient that the statement refers to a placebo, and if it therefore causes him to have a false belief, the patient's consent will not be valid. After all, the patient would be ignorant about the very object of consent, i.e. what he actually consents to (taking known analgesics versus placebos). And as this instance of ignorance would be due to physician behavior which fails to respect autonomy, the patient's consent to placebo treatment could no longer fulfill the purpose which informed consent is designed for, i.e. the protection of autonomy. Therefore, if placebos were administered according to Gligorov's proposal, the patients' consent, if based on a false belief, will be invalid.

\section{References}

Carson, T. 2010. Lying and Deception: Theory and Practice. Oxford University Press.

Gligorov, N. 2018. Telling the Truth about Pain: Informed Consent and the Role of Expectation in Pain Intensity. The American Journal of Bioethics - Neuroscience.

Greenspan, P. 2003. The Problem with Manipulation. American Philosophical Quarterly, 40, 155164.

O'Neill, O. 2002. Autonomy and Trust in Bioethics. Cambridge University Press.

This work is supported by the Arts and Humanities Research Council, UK. Grant number: AH/L503885/1 